\title{
A New Method for Testing Filtration Efficiency of Mask Materials Under Sneeze-like Pressure
}

\author{
LI XIAO $^{1}$, HIROSHI SAKAGAMI ${ }^{2}$ and NOBUHIKO MIWA ${ }^{3}$ \\ ${ }^{1}$ Department of Pharmacology, School of Life Dentistry at Tokyo, The Nippon Dental University, Tokyo, Japan; \\ ${ }^{2}$ Meikai University Research Institute of Odontology (M-RIO), Saitama, Japan; \\ ${ }^{3}$ Faculty of Life Sciences, Prefectural University of Hiroshima, Hiroshima, Japan
}

\begin{abstract}
Background: Sneezes produce many pathogencontaining micro-droplets with high velocities of 4.5-50.0 $\mathrm{m} / \mathrm{s}$. Face masks are believed to protect people from infection by blocking those droplets. However, current filtration efficiency tests can't evaluate masks under sneeze-like pressure. The goal of this study was to establish a method to evaluate the filtration efficiency of mask materials under extreme conditions. Materials and Methods: Efficiency of surgical masks, gauze masks, gauze, cotton, silk, linen and tissue paper on blocking micro-droplet sized starch particles (average $8.2 \mu \mathrm{m})$ and latex microspheres $(0.75 \mu \mathrm{m})$ with a velocity of $44.4 \mathrm{~m} / \mathrm{s}$ created by centrifugation was qualitatively analyzed by using imaging-based analysis. Results: The 4 layers of silk could block $93.8 \%$ of microspheres and $88.9 \%$ of starch particles, followed by the gauze mask $(78.5 \%$ of microspheres and $90.4 \%$ of starch particles) and the 2 layers of cotton (74.6\% of microspheres and $87.5-89.0 \%$ of particles). Other materials also blocked 53.2-66.5\% of microspheres and 76.4\%-87.9\% of particles except the 8 layers of gauze which only blocked $36.7 \%$ of particles. The filtration efficiency was improved by the increased layers of materials. Conclusion: Centrifugationbased filtration efficiency test not only compensates shortcomings of current tests for masks, but also offers a simple way to explore new mask materials during pandemics. Common mask materials can potentially provide protection against respiratory droplet transmission.
\end{abstract}

This article is freely accessible online.

Correspondence to: Li Xiao, M.D., Ph.D., Department of Pharmacology, School of Life Dentistry at Tokyo, The Nippon Dental University , Fujimi 1-9-20, Chiyoda-ku, Tokyo, 1020071, Japan. Tel: +81 332618772, Fax: +81 332648399, e-mail: xiaoli@tky.ndu.ac.jp

Key Words: Micro-droplets, pandemics, mask materials, filtration efficiency, SARS-CoV-2, sneeze velocity.
Severe acute respiratory syndrome coronavirus 2 (SARSCoV-2) causing respiratory infection, has rapidly spread worldwide. SARS-CoV-2 is primarily transmitted between people through respiratory droplets and small particle droplet nuclei (aerosols). Coughing and sneezing can produce airflows at high velocities containing countless microdroplets. Early studies showed that the velocity of a sneeze was about 46.0-50.0 m/s whereas recent studies demonstrated that the initial velocity of the micro-droplets in a sneeze was about 4.5-7.0 m/s $(1,2)$. Face masks, such as surgical masks could block micro-droplets $(>5 \mu \mathrm{m})$ and aerosols $(<5 \mu \mathrm{m})$ to prevent transmission of human coronaviruses and influenza viruses from symptomatic individuals (3). Bacterial filtration efficiency (BFE), viral filtration efficiency (VFE) and $0.1 \mu \mathrm{m}$ particle filtration efficiency (PFE) tests are well known for the materials used in the construction of medical face masks. These tests basically use aerosols of 0.1-3.0 $\mu \mathrm{m}$ latex spheres at a velocity below $0.25 \mathrm{~m} / \mathrm{s}$ which is much slower than that of a cough or sneeze (4). A fit test machine has not been designed for testing the filtration efficiency of masks at the air speed velocity of a sneeze or cough (instead is used for identifying leaks and ensuring the proper functioning of a face piece). A recent study reported that standard surgical masks were not able to block SARS-CoV-2 when COVID19 patients coughed (5). This evidence suggests that current tests for the filtration efficiency of masks probably can't offer appropriate standards for infectious disease prevention, especially while coughing and sneezing. Nevertheless, during pandemics, the global mask shortage is expected to be severe. Alternative respiratory protective materials, including common fabric materials such as $100 \%$ cotton from T-shirts, handkerchiefs, and scarves, have been recommended (6). Several studies have measured the filtration efficiency of those mask materials by PFE, a fit test machine or other methods at velocities below $0.25 \mathrm{~m} / \mathrm{s}$. However, the results from each study were quite different (7-10) and may cause confusion. Thus, it is urgent to develop a reproducible method for testing the filtration efficiency of mask materials while 

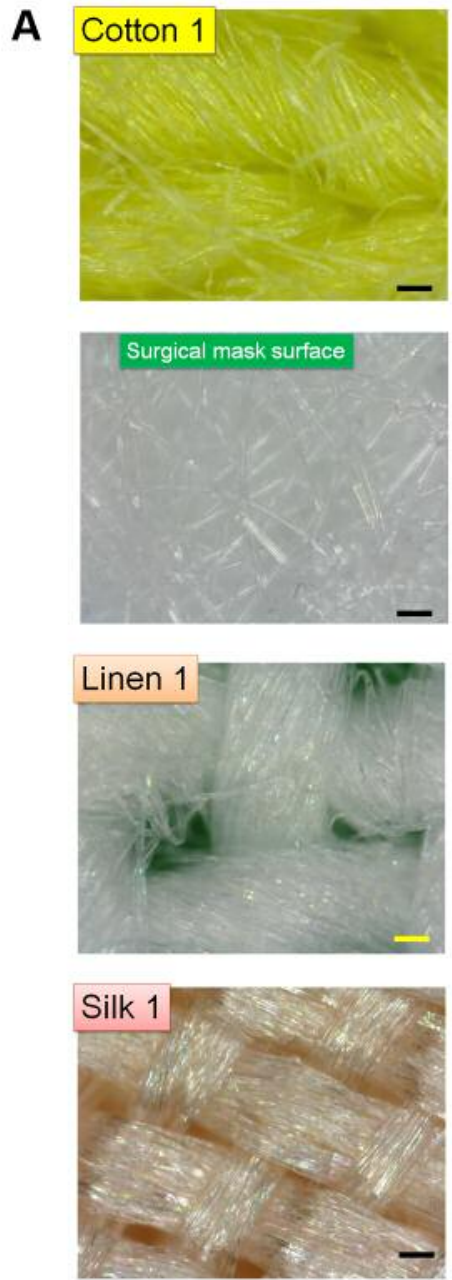
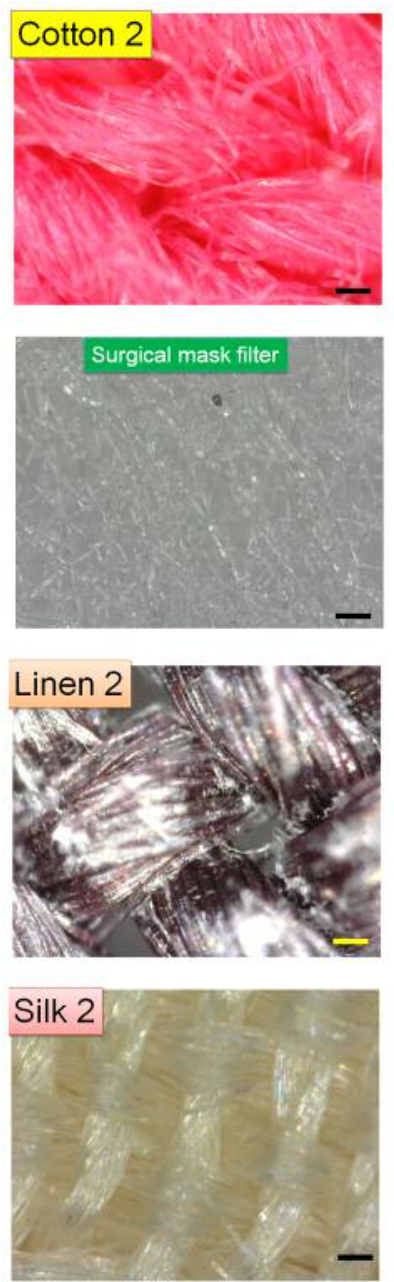
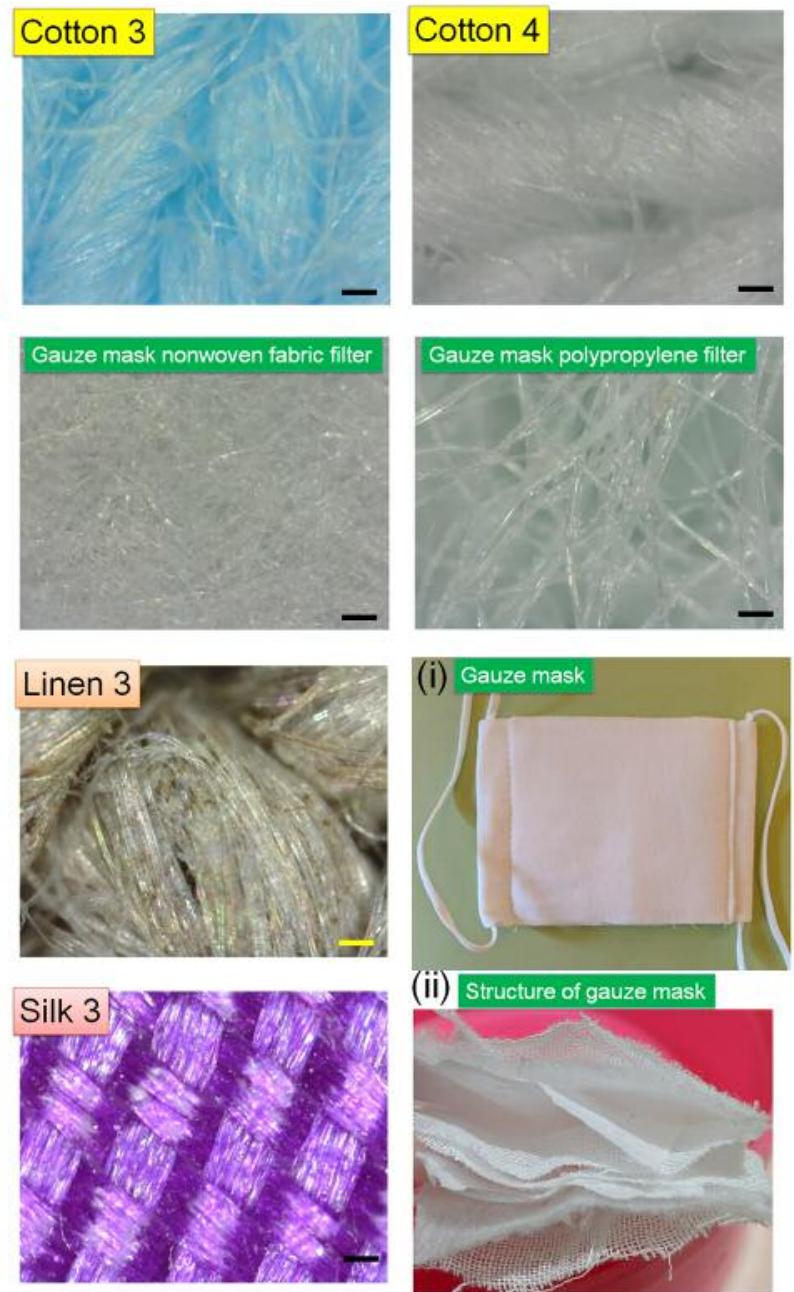

(ii) Structure of gauze mask

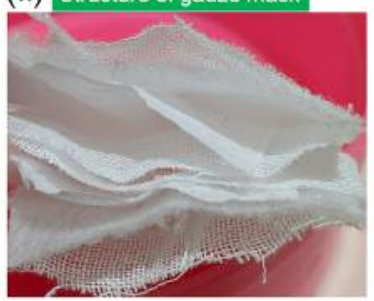

Figure 1. Continued sneezing or coughing. This study presents a simple way to test the filter performance of mask materials using microdroplets sized particles and microspheres with a challenged velocity of $44.4 \mathrm{~m} / \mathrm{s}$ created by centrifugation $(7,500 \mathrm{rpm})$.

\section{Materials and Methods}

Samples of mask materials. Samples included surgical masks, washed surgical masks, cotton gauze masks (containing 4 layers of cotton gauze, 2 layers of nonwoven fabric filter and 2 layers of polypropylene filter, washed), 4 types of cotton from T-shirts, 3 types of silk, 3 types of linen, tissue paper and cotton gauze. Surgical masks and cotton gauze masks were purchased from a Japanese drug store. Both of them have 99\% BFE. The surgical masks also passed PFE and VFE tests. The microstructures of mask materials were observed by both a digital microscope (AM4113ZT4, ANMO Electronics Corp., Taiwan) and a scanning electron microscope (SEM) (S-4000, HITACHI, Ltd. Tokyo).
Mask washing method. Both gauze masks and surgical masks were soaked in water with a neutral detergent for $10 \mathrm{~min}$. After rinsing with clean water 3-5 times, the masks were soaked in $1.5 \%$ chlorine bleach for another $10 \mathrm{~min}$. Then the masks were rinsed with clean water 3-5 times and air dried.

Starch solution preparation. One tea spoon of soft wheat flour (commercially available) was mixed with food coloring in $50 \mathrm{ml}$ of water. The solution was further completely mixed by voltex before being added onto each sample.

Microsphere solution preparation. One droplet of Fluoresbrite ${ }^{\circledR}$ YG Carboxylate Microspheres $0.75 \mu \mathrm{m}$ (Cat\#07766-10, Polysciences, Inc., Warrington, PA, USA) was added into $50 \mathrm{ml}$ of water to make an aqueous suspension. The solution was completely mixed by voltex before being added onto each sample.

Micro-particle filtration efficiency test. a) Starch particle filtration efficiency test. $300 \mu \mathrm{l}$ of starch solution was gently dropped on the 

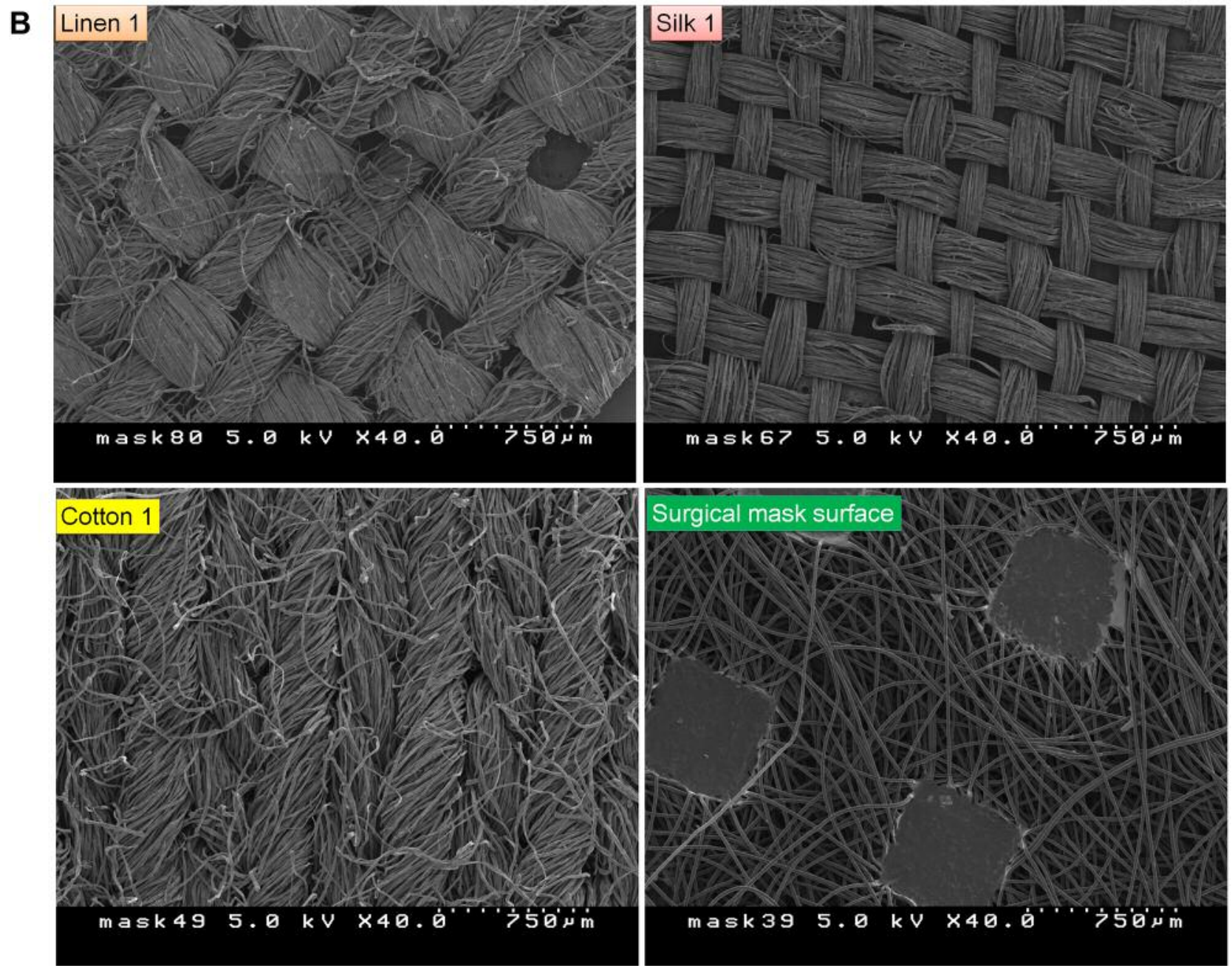

Figure 1. Microstructures of mask materials. A. Polarized microscopic images of mask materials including 4 types of 100\% cotton from T-shirts, surgical mask surface, surgical mask filter, gauze mask nonwoven fabric filter, gauze mask polypropylene filter, 3 types of linen, 3 types of silk. (i), commercial available cotton gauze mask, (ii) macrostructure of the cotton gauze mask. B. SEM images of some mask materials.

top of each sample which was set in the insert of a micro-tube [Figure 2(i)] and followed by centrifugation $(7,500 \mathrm{rpm}$, equaling $44.4 \mathrm{~m} / \mathrm{s}$ ) for $20 \mathrm{~s}$ to mimic the velocity of a sneeze. After centrifugation, the solution was mixed completely by pipetting. For the hydrophobic samples (surgical masks) or samples with thick layers, $100 \mu \mathrm{l}$ of the solution was applied on to the samples and centrifuged 6-7 s. This process was repeated three times. $25 \mu \mathrm{l}$ of the filtered solution was added onto a Tali ${ }^{\mathrm{TM}}$ Cellular Analysis Slide (T10794, Thermo Fisher Scientific, Tokyo) and analyzed by the Tali $^{\mathrm{TM}}$ Image Cytometer (Thermo Fisher Scientific, Tokyo).

b) Microsphere filtration efficiency test. $100 \mu \mathrm{l}$ of microsphere solution was added to surface of the samples and centrifuged in the same manner as described in the starch particle filtration efficiency test. The filtered solution was then mixed completely by pipetting A $1 \mu \mathrm{l}$ droplet of the solution was added onto a microscope glass slide and photographed with the EVOS ${ }^{\circledR}$ FL Cell Imaging System (Thermo Fisher Scientific, Tokyo, Japan). The green fluorescence intensity of each droplet was then analyzed by ImageJ (version $1.52 \mathrm{u}$, Wayne Rasband, National Institutes of Health, Bethesda, MD, USA).

c) Microsphere-capturing test. 50-100 $\mu \mathrm{l}$ of microsphere solution was added on top of the sample to make the materials wet throughout all the layers. After air-drying overnight, samples were mounted onto stubs and then coated with a thin layer of osmium in the osmium plasma coater (Neo osmium coater Neoc-AN, Meiwafosis Co., Ltd. Tokyo, Japan). The samples were examined using a scanning electron microscope (S-4000, HITACHI, Ltd. Tokyo, Japan).

Statistical analysis. Statistical analysis was carried out similarly to our previous report (11). All data, expressed as the mean $\pm \mathrm{SD}$, were analyzed statistically by GNU PSPP Statistical Analysis Software (version 0.8.2-gad9374) (https://www.gnu.org/software/pspp/) and EZAnalyze Excel-based tools (http://www.ezanalyze.com/). Oneway analysis of the variance was followed by post hoc analysis 
A

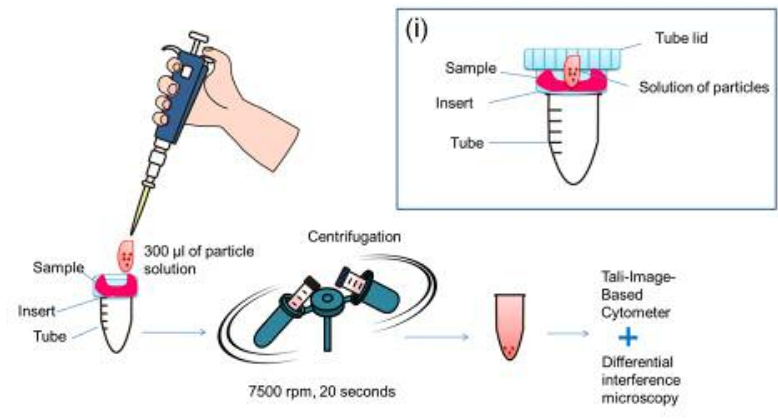

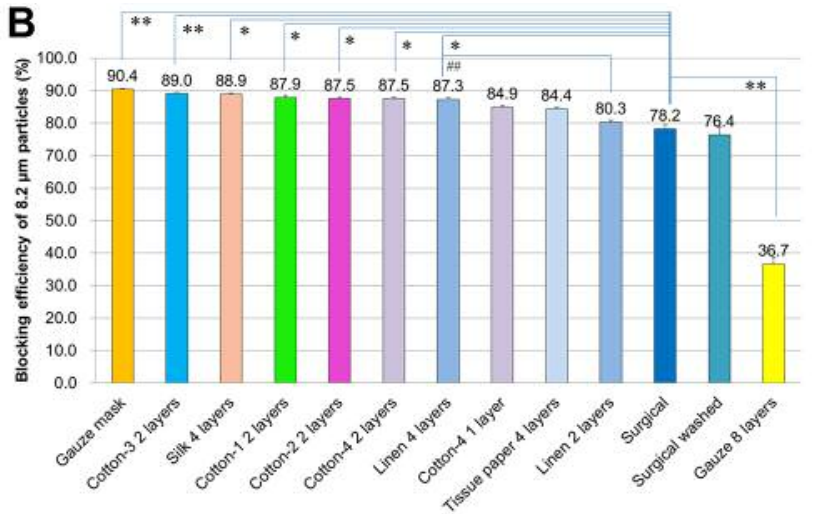
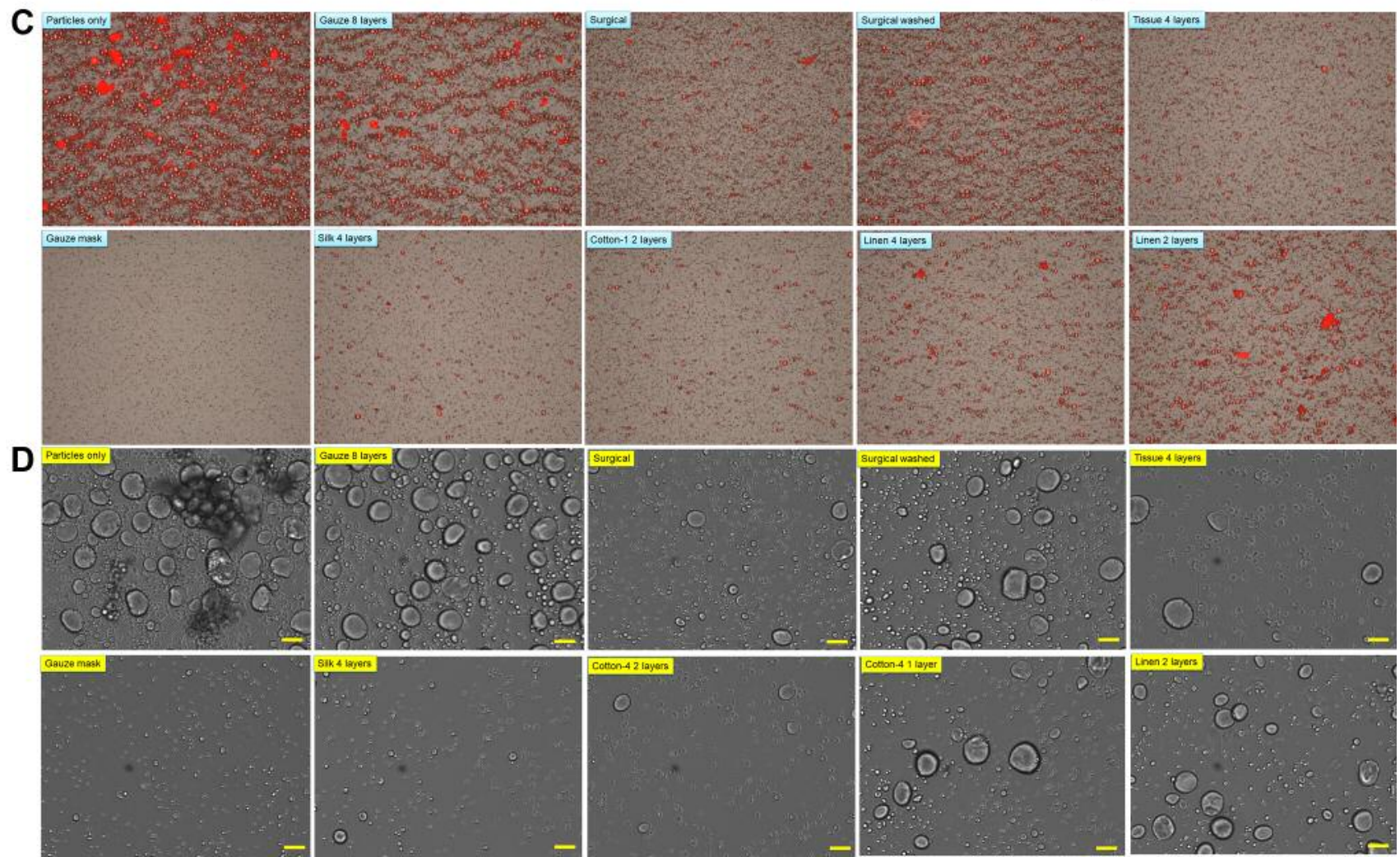

Figure 2. Efficiency of the mask materials for filtering micro-droplet-sized particles. The experimental steps are illustrated in A. (i) shows the setting of samples. B. Efficiency of mask materials on blocking starch particles was analyzed by Tali cytometer. The histograms represent three independent experiments. ${ }^{*} p<0.01, * p<0.05$, vs. surgical (surgical mask) (analyzed by both Tukey's test and Bonferroni Correction). C. Images of the filtered particles taken by Tali cytometer. D. Images of the starch particles taken by differential interference microscopy (400 $\times$ magnification). Scale $=50 \mu m$.

including Tukey's test and Bonferroni Correction. Statistical significance was considered when $p<0.05$. All experiments were repeated 3 times.

\section{Results}

Microstructures of mask materials. As shown in Figure 1A, the thickness of fibers and TPI (twists per inch) are all similar within each type of material. SEM images showed that the fibers of the cotton sample were closer to each other than in the linen and the silk samples. Because linen and silk naturally contain more water in their fibers than cotton, they probably lost more water during SEM sample preparation and observation (Figure 1B).

Starch particles filtration efficiency of mask materials. We used centrifugation $(7,500 \mathrm{rpm}$, equaling $44.4 \mathrm{~m} / \mathrm{s}$ for $20 \mathrm{~s})$ to simulate the velocity of a sneeze under extreme conditions, 
A

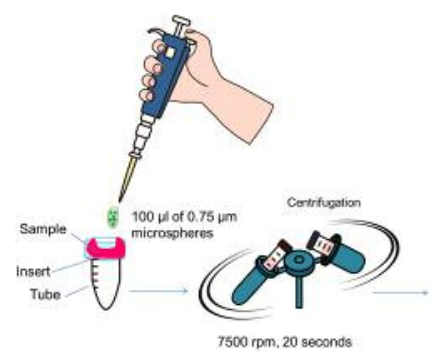

C
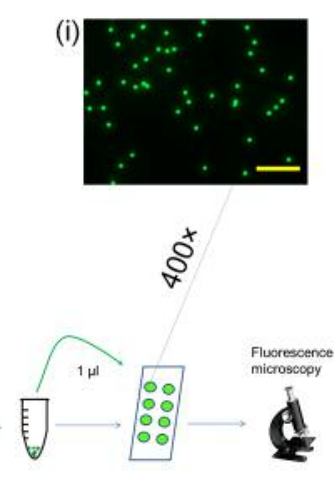

Silk 4 layers

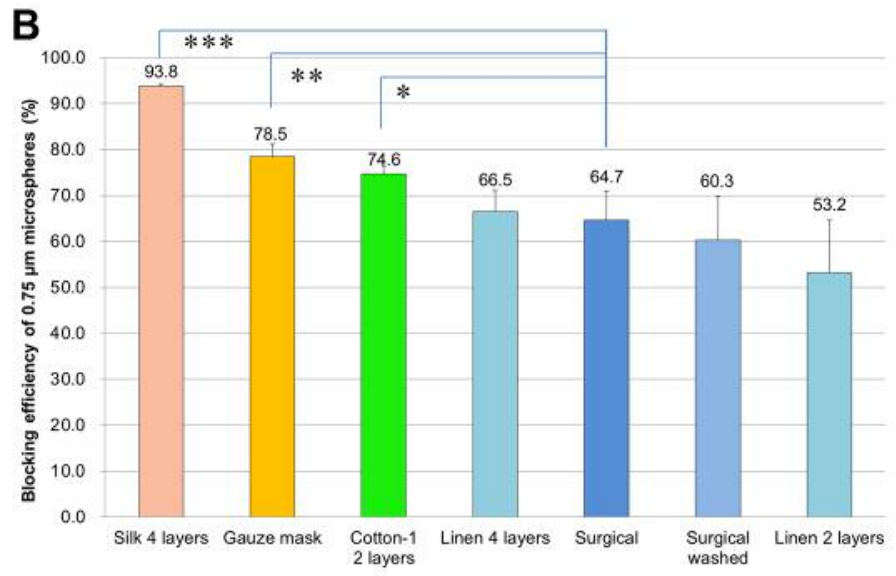

Gauze mask
Cotton-1 2 layers
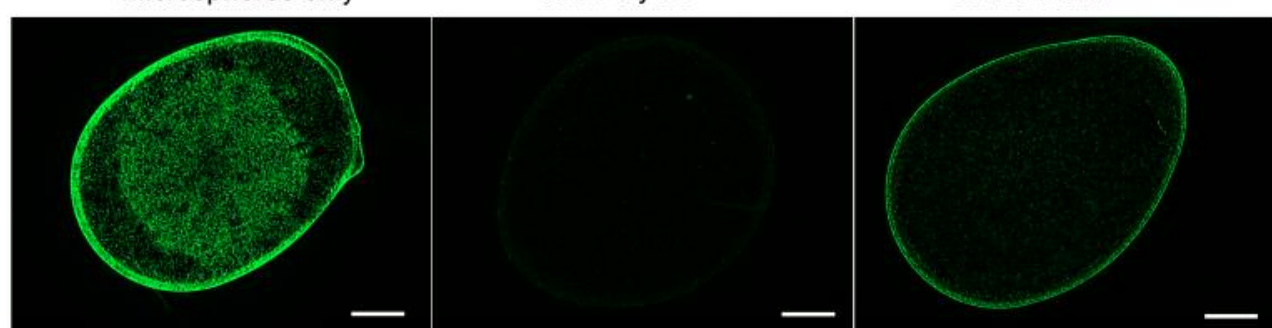

Linen 4 layers

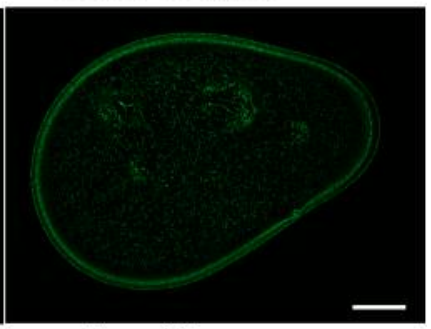

Surgical

Surgical washed

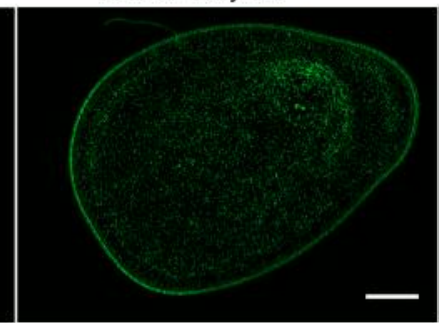

Linen 2 layers
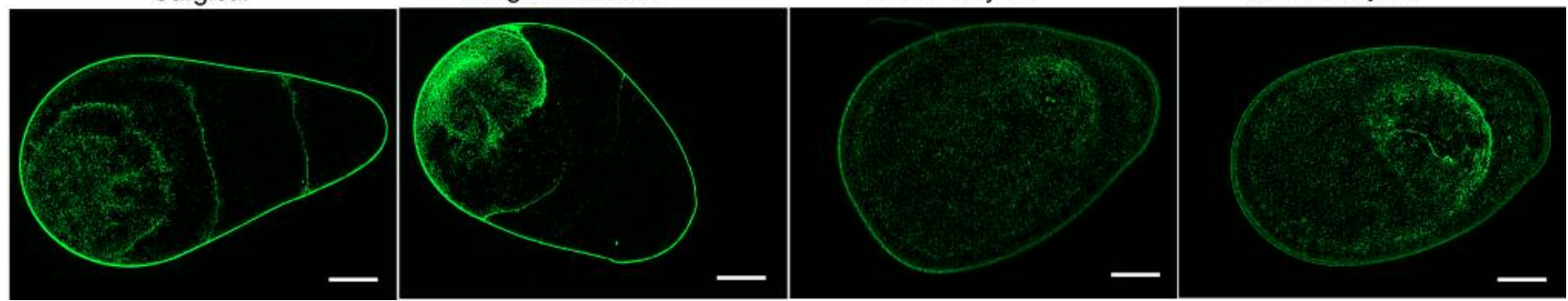

Figure 3. Efficiency of the mask materials for filtering aerosol-sized particles. The experimental steps are illustrated in A. (i) shows 400 times enlarged image of Fluoresbrite ${ }^{\circledR}$ YG Carboxylate Microspheres with a dimeter of $0.75 \mu \mathrm{m}$. Scale $=50 \mu \mathrm{m}$. B. Efficiency of mask materials in blocking microspheres was analyzed by EVOS ${ }^{\circledR}$ FL Cell Imaging System and ImageJ software. The histograms represent three independent experiments. $*^{* *} p<0.001,{ }^{*} p<0.01,{ }^{*} p<0.05$, vs. surgical mask (analyzed by both Tukey's test and Bonferroni Correction). C. Forty times enlarged images of the concentrated microspheres taken by EVOS fluorescence microscope. Scale $=500 \mu \mathrm{m}$.

and performed a series of experiments to test the filtration efficiency of mask materials. As shown in Figure 2D, the size of starch particles was about $0.7-70 \mu \mathrm{m}$. According to analysis from the Tali cytometer, their average size was about $8.2 \mu \mathrm{m}$ that is similar to micro-droplet-sized $(5-10 \mu \mathrm{m})$ particles $(12)$. Larger size particles can mimic bigger droplets $(>10 \mu \mathrm{m})$. Figure $2 \mathrm{~B}$ showed that during centrifugation, the cotton gauze mask could block $90.4 \%$ of the starch particles, followed by the 2 layers of cotton-3 $(89.0 \%)$ and the 4 layers of silk $(88.9 \%)$. Other materials also blocked $76.4 \%-87.9 \%$ particles except the 8 layers of gauze which only blocked $36.7 \%$. Notice that although the filtration efficiency of the gauze mask was higher than the 4 layers of silk, images from the Tali cytometer and microscopy showed that there were more small particles in the gauze mask filtered solution than that in the 4 layers of silk (Figure 2C and D).

Microsphere filtration efficiency of mask materials. We then tested the filtration efficiency of aerosol-sized particles. According to WHO's definition, the size of aerosols is below $5 \mu \mathrm{m}$ (12). Over $87 \%$ of particles in exhaled breath of influenza-infected patients were under $1 \mu \mathrm{m}$ (13). Therefore, we used the latex microspheres $(0.75 \mu \mathrm{m})$ to mimic aerosols. Figure 3(i) showed that the microspheres were even sized particles emitting the green fluorescence. Figure 3C showed that $1 \mu \mathrm{l}$ of the concentrated microspheres made spots with 

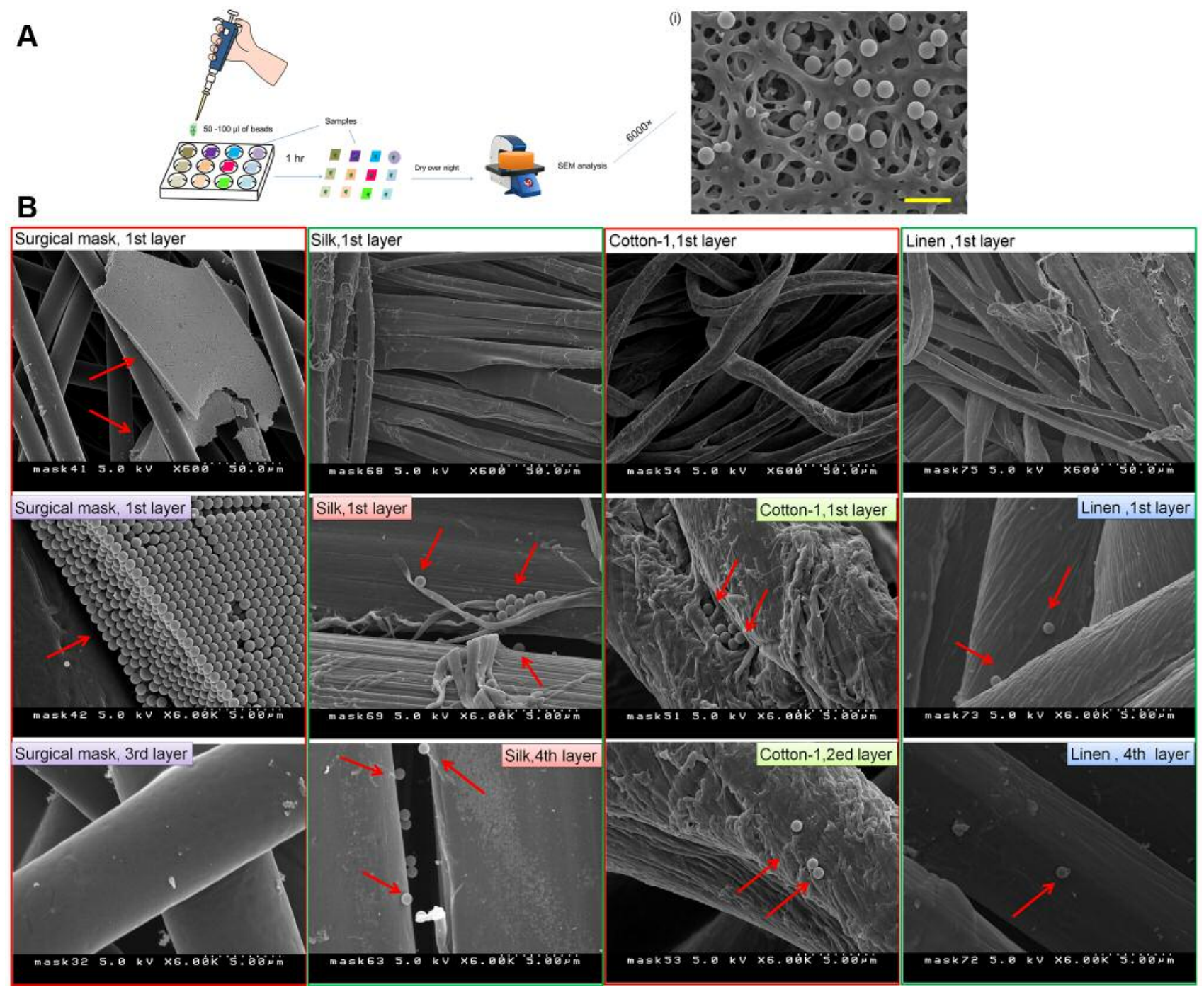

Figure 4. Microsphere-capturing ability of mask materials. The experimental steps are illustrated in A. (i) presents a SEM image of the microspheres on a hydrophilic PVDF membrane filter (0.22 $\mu \mathrm{m}$ pore size), scale bar $=2 \mu \mathrm{m}$. B. SEM images of the microspheres captured by the mask materials (shown by red arrows).

green fluorescence. The green fluorescence intensity level of the spots represents the number of microspheres existing in the $1 \mu$ solution. Because these microspheres were too small to measure by the Tali cytometer or flow cytometry, we analyzed the green fluorescence intensity of each spot to quantify the microspheres blocking rate of mask materials using the ImageJ software. As shown in Figure 3B and C, all the mask materials could block the microspheres at different degrees. The top three microspheres blocking materials were the 4 layers of silk, the gauze mask and the 2 layers of cotton-1 (cotton-1 showed average filtration efficiency among cotton 1-4 in the starch particles test, therefore we chose it for this experiment). They all showed significantly higher blocking ability than the surgical mask. Because silk is electrostatically charged, the 4 layers of silk showed the highest blocking rate (93.8\%) of the microspheres. Cotton and linen with multiple layers were significantly better than a single or fewer layers. Washed surgical masks showed lower filtration efficiency than the new surgical masks.

Microsphere-capturing ability of mask materials. Lastly, we tested the microsphere-capturing ability of materials without centrifugation by using SEM (Figure 4). This experiment simulated a situation in which the micro-droplets are spewed onto the surface of a mask. Figure 4(i) showed that the microspheres did not penetrate the $0.22 \mu \mathrm{m}$ filter. Because the surface of surgical masks is hydrophobic, $50 \mu \mathrm{l}$ of microspheres did not pass through the surface without 
centrifugation. The microspheres accumulated on the fibers of the mask surface, and were not seen in the third layer. Meanwhile, the solution of microspheres penetrated the layers of silk, the cotton and the linen samples. Microspheres were observed both on their first and last layers, suggesting that these materials could capture those microspheres. Notice that some microspheres presented on the edge of the silk fibers suggesting that silk fibers probably electrostatically captured those microspheres.

\section{Discussion}

Previous studies suggested that the filtration efficiency of masks is basically affected by particle size and filtration velocity (14). Centrifugation can easily produce enough force to separate the particles inside a solution at high velocities. As we demonstrate in Figure 2(i), with an insert placed in the tube, it is simple to check the filtration efficiency of materials by centrifugation. Unlike direct force produced by negative pressure, this method does not break the fibers of the testing materials even at a challenged velocity. The mask materials we used in this study were similar to a previous study in which the researchers evaluated the capacity of homemade masks to block bacterial and viral aerosols by using a fit test machine. Their results showed that $100 \%$ cotton from $\mathrm{T}$-shirts was the best choice for homemade masks although its efficiency in blocking transmission was 3 times lower than that of surgical masks (9). A recent study also checked the filtration efficiency of common fabrics at two different airflows: 35 $\mathrm{L} / \mathrm{min}$ and $\sim 90 \mathrm{~L} / \mathrm{min}$. The data showed that some cotton fabrics and the 4 layers of silk exhibited better or equal efficiency to that of N95 or surgical masks on blocking aerosol particles $(10 \mathrm{~nm}$ to $6.0 \mu \mathrm{m})$. The filtration efficiencies were improved by the increased TPI or layers of fabrics (10). Our data showed similar results; the 4 layers of silk, the cotton gauze masks, and the 2 layers of cotton from T-shirts could efficiently block $0.75 \mu \mathrm{m}$ microspheres and $8.2 \mu \mathrm{m}$ starch particles under a sneeze-like pressure. Their blocking efficiency was significantly better than the surgical masks. Evidence showed that surgical masks could not efficiently filter particles under a size of $3.1 \mu \mathrm{m}$ (4). These phenomena possibly occur because the intervals of fibers in surgical masks are much looser than in cotton and silk (Figure 1B and 4B); micro-particles can easily pass through the surgical mask layers at the velocity of a sneeze. However, since surgical masks have hydrophobic and electrocharged fibers, they can offer fluid resistance and electrostatically capture micro-particles. Interestingly our data showed that the 4 layers of tissue paper could block $84.4 \%$ of starch particles, which was significantly more efficient than surgical masks. It has been reported that infectious SARS-CoV-2 could be detected on the inner and outer layers of a surgical mask for 4-7 days whereas they could not be detected on tissue paper after $3 \mathrm{~h}$ (15). Therefore, putting several layers of tissue paper inside the surgical mask could offer more protection than a surgical mask alone. Washed surgical masks lost their hydrophobicity and static electricity. They showed lower blocking rates of starch particles and microspheres.

Previous studies showed that cotton fabric could reduce virus titer $(16,17)$ suggesting that cotton is a suitable material for masks. Linen has been used in hospital textiles due to its ability to disperse heat and evaporate moisture. Our data showed that 2 layers of linen could block $53.2 \%$ of the microspheres and $80.3 \%$ of the starch particles suggesting that it has the ability to block some microdroplets. Taken together, we recommend people to make masks with 1) 2 layers of $100 \%$ cotton from T-shirts, 2) 4 layers of silk or 3 ) a combination of linen/cotton or silk/linen or silk/cotton (using a wire around the nose could make the masks fit well). However, none of these materials could block particles totally, and even a small percentage of the micro-droplets and aerosols that passed through the masks is enough to cause infection. Therefore keeping a social distance, avoiding crowds and frequently airing rooms are very important.

However, this study has some limitations. Centrifugationproduced velocity only partially represents the velocity of a cough or a sneeze. The dynamics of particles in fluid and in air are also different. It would be best if this study had used human subjects to test the filtration efficiency of masks while coughing and sneezing. It has been reported that digital high-vision and high-speed video systems could quantitatively analyze micro-droplets while coughing or sneezing $(18,19)$. This technique could be applied for testing the filtration efficiency of masks.

\section{Conclusion}

To our knowledge, this is the first report to use centrifugation to test the filtration efficiency of mask materials under sneeze-like pressure. This method not only compensates for shortcomings of PFE, VFE and BFE tests, but also offers a simple way to explore new materials for manufacturing masks during pandemics.

\section{Conflicts of Interest}

The Authors declare no competing financial interests regarding this study.

\section{Authors' Contributions}

L. X. designed and performed experiments, analyzed data and wrote the paper; H. S analyzed data and gave conceptual advices; N.M. provided materials, analyzed data and proofread the manuscript. 


\section{Acknowledgements}

The present study was supported in part by a Grant-in-Aid for AntiAging Scientific Research \#1517 by Japanese Center for AntiAging MedSciences which was authenticated by Hiroshima Prefecture Government as a non-profitable organization corporation, Hiroshima, Japan to XL. The author would like to thank Fusako Mitsuhashi for her technical support. The authors also appreciate Nathaniel Green's proofreading.

\section{References}

1 Xie X, Li Y, Chwang AT, Ho PL and Seto WH: How far droplets can move in indoor environments--revisiting the Wells evaporation-falling curve. Indoor Air 17(3): 211-225, 2007. PMID: 17542834. DOI: 10.1111/j.1600-0668.2007.00469.x

2 Nishimura H, Sakata S and Kaga A: A new methodology for studying dynamics of aerosol particles in sneeze and cough using a digital high-vision, high-speed video system and vector analyses. PLoS One 8(11): e80244, 2013. PMID: 24312206. DOI: $10.1371 /$ journal.pone. 0080244

3 Leung NHL, Chu DKW, Shiu EYC, Chan KH, McDevitt JJ, Hau BJP, Yen HL, Li Y, Ip DKM, Peiris JSM, Seto WH, Leung GM, Milton DK and Cowling BJ: Respiratory virus shedding in exhaled breath and efficacy of face masks. Nat Med 26: 676680, 2020. PMID: 32371934. DOI: 10.1038/s41591-020-0843-2

4 Oberg $\mathrm{T}$ and Brosseau LM: Surgical mask filter and fit performance. Am J Infect Control 36(4): 276-282, 2008. PMID: 18455048. DOI: 10.1016/j.ajic.2007.07.008

5 Bae S, Kim MC, Kim JY, Cha HH, Lim JS, Jung J, Kim MJ, Oh DK, Lee MK, Choi SH, Sung M, Hong SB, Chung JW and Kim SH: Effectiveness of Surgical and Cotton Masks in Blocking SARS-CoV-2: A Controlled Comparison in 4 Patients. Ann Intern Med M20-1342, 2020. PMID: 32251511. DOI: $10.7326 / \mathrm{M} 20-1342$

6 Use of cloth face coverings to help slow the spread of COVID19. COVID-19. Centers of Disease Control and Prevention. Available at: https://www.cdc.gov/coronavirus/2019-ncov/ prevent-getting-sick/diy-cloth-face-coverings.html [Last accessed April 30, 2020]

7 Cooper DW, Hinds WC, Price JM, Weker R and Yee HS: Common materials for emergency respiratory protection: leakage tests with a manikin. Am Ind Hyg Assoc J 44(10): 720-726, 1983. PMID: 6650392. DOI: 10.1080/15298668391405634

8 Rengasamy S, Eimer B and Shaffer RE: Simple respiratory protection--evaluation of the filtration performance of cloth masks and common fabric materials against 20-1000 nm size particles. Ann Occup Hyg 54(7): 789-798, 2010. PMID: 20584862. DOI: $10.1093 /$ annhyg/meq044
9 Davies A, Thompson KA, Giri K, Kafatos G, Walker J and Bennett A: Testing the efficacy of homemade masks: would they protect in an influenza pandemic? Disaster Med Public Health Prep 7(4): 413-418, 2013. PMID: 24229526. DOI: $10.1017 / \mathrm{dmp} .2013 .43$

10 Konda A, Prakash A, Moss GA, Schmoldt M, Grant GD and Guha S: Aerosol filtration efficiency of common fabrics used in respiratory cloth masks. ACS Nano acsnano.0c03252, 2020. PMID: 32329337. DOI: 10.1021/acsnano.0c03252

11 Xiao L, Saiki C and Okamura H: Oxidative stress-tolerant stem cells from human exfoliated deciduous teeth decrease hydrogen peroxide-induced damage in organotypic brain slice cultures from adult mice. Int J Mol Sci 20(8): 1858, 2019. PMID: 30991705. DOI: 10.3390/ijms20081858

12 Infection Prevention and Control of Epidemic-and Pandemic-Prone Acute Respiratory Infections in Health Care. World Health Organization; 2014. Available at: www.who.int/csr/bioriskreduction/ infection_control/publication/en [Last accessed April 30, 2020]

13 Fabian P, Mcdevitt JJ, Dehaan WH, Fung RO, Cowling BJ, Chan KH, Leung GM and Milton DK: Influenza virus in human exhaled breath: an observational study. PloS One 3(7): e2691, 2008. PMID: 18628983. DOI: 10.1371/journal.pone.0002691

14 Mostofi R, Wang B, Haghighat F, Bahloul A and Jaime L: Performance of mechanical filters and respirators for capturing nanoparticles--limitations and future direction. Ind Health 48(3): 296-304, 2010. PMID: 20562505. DOI: 10.2486/indhealth.48.296

15 Chin AWH, Chu JTS, Perera MRA, Hui KPY, Yen HL, Chan MCW, Peiris M and Poon LLM: Stability of SARS-CoV-2 in different environmental conditions. Lancet Microbe, 2020. DOI: 10.1016/S2666-5247(20)30003-3

16 Sidwell RW, Dixon GJ and McNeil E: Quantitative studies on fabrics as disseminators of viruses. I. Persistence of vaccinia virus on cotton and wool fabrics. Appl Microbiol 14(1): 55-59, 1966. PMID: 5953019.

17 Dixon GJ, Sidwell RW and McNeil E: Quantitative studies on fabrics as disseminators of viruses. II. Persistence of poliomyelitis virus on cotton and wool fabrics. Appl Microbiol 14(2): 183-188, 1966. PMID: 4289622.

18 Nishimura H, Sakata S and Kaga A: A new methodology for studying dynamics of aerosol particles in sneeze and cough using a digital high-vision, high-speed video system and vector analyses. PLoS One 8(11): e80244, 2013. PMID: 24312206. DOI: 10.1371 /journal.pone.0080244

19 Bourouiba L: Turbulent gas clouds and respiratory pathogen emissions: Potential implications for reducing transmission of COVID-19. JAMA, 2020. PMID: 32215590. DOI: 10.1001/ jama.2020.4756

Received May 21, 2020

Revised May 25, 2020

Accepted May 26, 2020 